\title{
Dielectric-constant evaluation from microstructures
}

\author{
Hongru Ma, ${ }^{*}$ Baoshe Zhang, Wing Yim Tam, and Ping Sheng \\ Department of Physics, The Hong Kong University of Science and Technology, Clear Water Bay, Kowloon, Hong Kong, China
}

(Received 4 June 1999; revised manuscript received 14 October 1999)

\begin{abstract}
We present an accurate, efficient algorithm for calculating the effective dielectric constant $\bar{\varepsilon}$ of periodic two-component composites with arbitrary unit-cell microstructures. The algorithm is based on a formulation of the relevant equations in the Bergman-Milton representation of $\bar{\varepsilon}$ that enables the use of the fast Fourier transform in their solution, coupled with the sum rules of the geometric spectral function. Our approach accurately reproduces known results, such as the two-dimensional checkerboard problem, and yields excellent agreement with experimentally measured results on random configurations of metallic spheres.
\end{abstract}

\section{INTRODUCTION}

Calculating the effective dielectric properties of heterogeneous materials is a classical problem in physics and mathematics that dates back to the early works by Rayleigh ${ }^{1}$ and Clausius-Mossotti. ${ }^{2}$ Its importance stems from one of the basic aims of materials research: relating (predicting) the macroscopic optical and transport properties of inhomogeneous systems to (from) those of atomic or mesoscopic constituents. In the past two decades, there has been a strong revival of interest in composite problems focused mostly on the critical transport and geometric behaviors close to the percolation threshold. For the study of such characteristics it has been shown that the use of discretized models is sufficient to yield the universal critical exponents and critical geometric properties. ${ }^{3}$ Away from the threshold, however, the nonuniversal character of the dielectric problem means the actual microstructures have to be taken into account, and thus the full difficulty of the classical problem returns. Various effective medium theories have been formulated to calculate the effective dielectric constant $\bar{\varepsilon}$ of composites with different types of microstructures, e.g., the Maxwell-Garnett theory (for the dispersion microstructure), ${ }^{4}$ the Bruggeman's selfconsistent theory (for the symmetric microstructure), ${ }^{5}$ the theory for the granular microstructures, ${ }^{6}$ or the differential effective theory, originally due to Bruggeman (for the hierarchical microstructure). ${ }^{5,7}$ However, all effective medium theories fail in terms of accurately relating an arbitrary microstructure to its complex effective dielectric constant. In view of the recent developments in microtomography, ${ }^{8}$ which can routinely generate three-dimensional (3D) microstructural data with microns resolution, the lack of a viable theoretical approach to this problem represents a serious gap in our ability to translate microstructural data into meaningful dielectric information.

In this work, we present an accurate and efficient algorithm for calculating the complex effective dielectric constant of periodic two-component composites with arbitrary unit-cell microstructures. Our approach is based on a reformulation of the relevant equations in the Bergman-Milton representation of $\bar{\varepsilon},{ }^{9}$ that enables the use of the fast Fourier transform (FFT) in their solution process. A direct consequence is that $10^{6}-10^{7}$. Fourier components can be easily handled (on a workstation) in the calculation of the geomet- ric spectral function $\mu(\chi)$. When this FFT approach is coupled with the exact sum rules for $\mu(\chi)$, accurate evaluation of $\bar{\varepsilon}$ becomes possible as illustrated below, even when the dielectric contrast between the two components, $\left|\varepsilon_{1} / \varepsilon_{2}\right|$, approaches $\infty$ and touching microgeometries are present. In particular, we show that our approach accurately reproduces the $\mu(\chi)$ for the 2D checkerboard problem, as well as yields good agreement with experimentally measured results on random configurations. These results imply that even for the random composite case, as long as the geometric correlation length is finite and not too large, our approach can yield reasonably accurate results by using unit cells larger than a typical correlated volume.

\section{FORMULATION}

The basic task of effective dielectric constant calculation is the solution of the equation

$$
\nabla \cdot \varepsilon(\vec{r}) \nabla \phi=0
$$

under the imposed condition of an external potential $\phi_{0}=z$ (unit electric field in the $z$ direction). By expressing $\varepsilon(\vec{r}) / \varepsilon_{2}=1-[\eta(\vec{r}) / s]$, where $s=\varepsilon_{2} /\left(\varepsilon_{2}-\varepsilon_{1}\right)$ is the only material parameter in the problem and $\eta(\vec{r})$ is the characteristic function, defined as $\eta(\vec{r})=1$ inside component 1 and zero otherwise, Eq. (1) becomes

$$
\nabla^{2} \phi=\frac{1}{s} \nabla \cdot \eta(\vec{r}) \nabla \phi .
$$

A formal solution of Eq. (2) is possible by using the Green's function of the Laplacian, $G\left(\vec{r}, \vec{r}^{\prime}\right)=1 / 4 \pi\left|\vec{r}-\vec{r}^{\prime}\right|$ :

$$
|\phi\rangle=s(s-\Gamma)^{-1}|z\rangle,
$$

where the operator $\Gamma$ is given by

$$
\Gamma=\int d \vec{r}^{\prime} \eta\left(\vec{r}^{\prime}\right) \nabla^{\prime} G\left(\vec{r}-\vec{r}^{\prime}\right) \cdot \nabla^{\prime} .
$$

It should be noted that $\Gamma$ is a purely geometric operator as it depends only on the microstructure of the system. Under the definition of the inner product

$$
\langle\phi \mid \psi\rangle=\int \eta\left(\vec{r}^{\prime}\right) \nabla^{\prime} \phi\left(\vec{r}^{\prime}\right) \cdot \nabla^{\prime} \psi\left(\vec{r}^{\prime}\right) d \vec{r}^{\prime},
$$


the operator $\Gamma$ is Hermitian, with eigenvalues confined in the interval $[0,1]$. From Eqs. (2)-(5), it is straightforward to obtain the Bergman-Milton representation of $\bar{\varepsilon}:^{9}$

$$
\frac{\bar{\varepsilon}}{\varepsilon_{2}}=1-\int \frac{\mu(\chi)}{s-\chi} d \chi=1-F(s),
$$

where $\mu(\chi)=|\langle z \mid \chi\rangle|^{2}$ is the geometric spectral function, and $|\chi\rangle$ denotes the eigenfunction of the $\Gamma$ operator with eigenvalue $\chi$. From Eq. (6) it is clear that if the spectral function is known accurately, the value of $\bar{\varepsilon}$ may be simply obtained even in the limit of high-contrast ratio, i.e., $\left|\varepsilon_{1} / \varepsilon_{2}\right| \rightarrow \infty$. Our goal is thus the evaluation of $\mu(\chi)$ from microstructure data.

\section{THE SOLUTION APPROACH}

For systems with arbitrary periodic microstructures, it is advantageous to seek the solution of Eq. (3) in the Fouriertransformed $\vec{k}$ space. To this end, we first express $\psi(\vec{r})$ $=\phi(\vec{r})-z$ as the periodic component of the potential that deviates from the uniform field case. By writing $a_{k}=i k \psi_{\vec{k}}$, Eq. (3) may be expressed as ${ }^{10}$

$$
s a_{\vec{k}}-\sum_{\vec{q} \neq 0} \Gamma_{\vec{k} \vec{q}} a_{\vec{q}}=\hat{k} \cdot \hat{e}_{z} \eta(\vec{k}),
$$

where $\Gamma_{\vec{k}} \vec{q}=\hat{k} \cdot \hat{q} \eta(\vec{k}-\vec{q})$ is the expression for the $\Gamma$ operator in the wave vector space, and the overhat denotes a unit vector. We wish to seek the eigenvalues and eigenfunctions of $\Gamma$ by using the Lanczos recursion method. ${ }^{11}$ That is, let $|0\rangle=(1 / \alpha)|z\rangle$, with $\alpha$ being the normalization constant, and define

$$
\Gamma|i\rangle=b_{i+1}|i+1\rangle+a_{i}|i\rangle+b_{i-1}|i-1\rangle,
$$

$\left(b_{0}=0\right)$, where the $a_{i}$ 's and the $b_{i}$ 's are determined by the conditions of orthogonality and normalization. Under this recursive transformation, $\Gamma$ is tridiagonal and thus its eigenvectors and eigenvalues can be easily calculated. By denoting $|\bar{\chi}\rangle$ as the eigenfunction with the uniform electric field component subtracted off, one can express $|\bar{\chi}\rangle=\Sigma_{i} C_{i}^{\chi}|i\rangle$. Since the spectral function involves only $C_{0}^{\chi}$, it is easy to show from Eqs. (6) and (7) that

$$
F(s)=\frac{1}{s}\left[p+\alpha^{2} \sum_{\chi} \frac{\left|C_{0}^{\chi}\right|^{2}}{s-\chi}\right],
$$

where $p$ denotes the volume fraction of component 1 . From Eq. (9) it is simple to find the spectral function as $\chi \mu(\chi)$ $=\alpha^{2}\left|C_{0}^{\chi}\right|^{2}$.

In the recursion approach, the most time-consuming operation is the matrix multiplication. Since $\Gamma$ is a full-matrix, one 3D matrix multiplication requires $N^{6}$ operations, where $N$ is the number of Fourier components along one direction. However, by defining two states, $\left|i_{a}\right\rangle$ and $\left|i_{b}\right\rangle$, as

$$
\begin{aligned}
\left\langle\vec{q} \mid i_{a}\right\rangle & =0 \quad \text { if } \quad \vec{q}=0, \\
& =\frac{1}{q}\langle\vec{q} \mid i\rangle \quad \text { otherwise, }
\end{aligned}
$$

$$
\left\langle\vec{q} \mid i_{b}\right\rangle=q\langle\vec{q} \mid i\rangle,
$$

the matrix multiplication may be expressed as a convolution operation:

$$
\begin{aligned}
\sum_{\vec{q} \neq 0} \Gamma_{\vec{k} \vec{q}}\langle\vec{q} \mid i\rangle= & \sum_{\vec{q}}\left\{\frac{k}{2} \eta(\vec{k}-\vec{q})\left\langle\vec{q} \mid i_{a}\right\rangle+\frac{1}{2 k} \eta(\vec{k}-\vec{q})\left\langle\vec{q} \mid i_{b}\right\rangle\right. \\
& \left.-\frac{1}{2 k}(\vec{k}-\vec{q})^{2} \eta(\vec{k}-\vec{q})\left\langle\vec{q} \mid i_{a}\right\rangle\right\} .
\end{aligned}
$$

That means the matrix multiplication can now be performed with the help of FFT, thus reducing the total operations count in one $3 \mathrm{D}$ matrix multiplication to $4.3 N^{4}+3 N^{3}$ at the most. For $N=100$, that translates to a gain of $10^{3}$ in terms of efficiency, thereby transforming a nearly impossible problem to one that is calculable.

The increased number of microstructure Fourier components, made possible by the recursive FFT method, enables the accurate evaluation of $\mu(\chi)$ away from the point $\chi=0$. However, near $\chi=0$, which dominates the behavior of $\bar{\varepsilon}$ in the limit of $\left|\varepsilon_{1} / \varepsilon_{2}\right| \rightarrow \infty$, this increase of calculational capability in itself is still insufficient to accurately determine the correct quantitative behavior of $\mu(\chi)$. Instead, we note that $\mu(\chi)$ must satisfy the sum rules, which relate the values of $\mu(\chi)$ near $\chi=0$ to the integrated value away from $\chi=0$. Thus the accurate determination of the spectral function away from $\chi=0$ may be used to advantage in evaluating $\mu(\chi)$ near $\chi=0$.

\section{APPLICATION}

In this section we illustrate our solution approach through different classes of examples.

(1) Simple cubic array of closely spaced spheres. Let the sphere dielectric constant be $\varepsilon_{1}$ and that of the matrix be $\varepsilon_{2}$. The sphere radius is denoted by $R$ and the lattice constant by $a$, where $a / 2 R=1.01$, i.e., the spheres are closely spaced. The Fourier transform of the characteristic function is given by

$$
\eta(\vec{k})=\frac{4 \pi}{(k a)^{3}}[\sin (k R)-k R \cos (k R)] .
$$

We have carried out the recursive-FFT calculation of the spectral function $\mu(\chi)$ with $N=135$, i.e., about $2.5 \times 10^{6}$ Fourier components. In this case, since the spheres are separated, the spectral function is known to have a gap extending from $\chi=0$ to some finite value. In order to clearly identify this gap, we first broaden each eigenvalue with a suitably chosen width, e.g., 0.01. The peaks of the resulting continuous spectrum are then identified by checking the second derivative of the spectrum. The whole spectrum is fit by $\mu(\chi)=\sum_{i} A_{i} g\left(\chi, \chi_{i}, a_{i}\right)$, where $g\left(\chi, \chi_{i}, a_{i}\right)=\exp \left[-\left(\chi-\chi_{i}\right)^{2} /\right.$ $\left.a_{i}^{2}\right] / a_{i} \sqrt{\pi}$ is a Gaussian. The lowest eigenvalue and its weight, $\chi_{1}$ and $A_{1}$, are then slightly adjusted so that the two sum rules, the zeroth moment $\Sigma_{i} A_{i}=p$ and the first moment $\Sigma_{i} \chi_{i} A_{i}=p(1-p) / 3$, are satisfied. The effective dielectric constant is given by Eq. (6) with

$$
F(s)=\sum_{i} \frac{A_{i}}{s-\chi_{i}}
$$




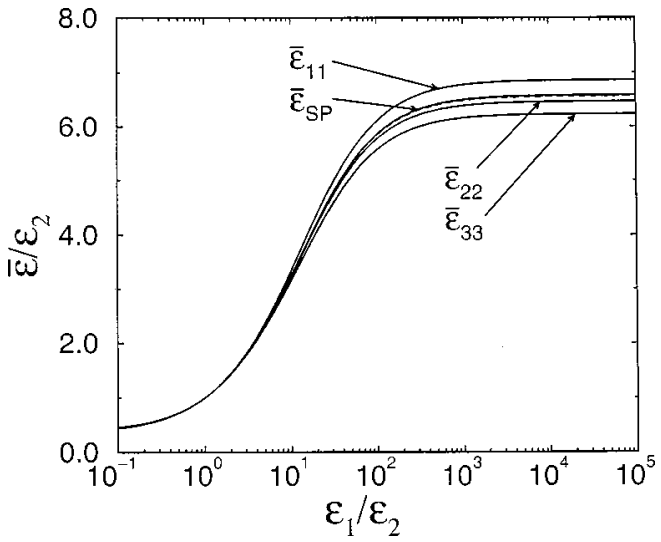

FIG. 1. Calculated effective dielectric constants for a simple cubic array of closely-spaced spheres $\left(\bar{\varepsilon}_{\mathrm{SP}}\right)$ and an orthorhombic array of closely-spaced spheroids $\left(\bar{\varepsilon}_{11}>\bar{\varepsilon}_{22}>\bar{\varepsilon}_{33}\right.$ in the three orthogonal directions), plotted as a function of $\varepsilon_{1} / \varepsilon_{2}$, assumed to be real in the present case. Parameters are given in the text. The dashed line is the accurate result calculated from the spherical basis functions in real space. Maximum deviation of $0.3 \%$ is seen in the saturation value of $\bar{\varepsilon}_{\mathrm{SP}}$, achieved in the limit of $\varepsilon_{1} / \varepsilon_{2} \rightarrow \infty$.

The calculated $\bar{\varepsilon}$ is plotted in Fig. 1 as a function of $\varepsilon_{1} / \varepsilon_{2}$, taken to be real in this case. Here the dashed line is calculated by a real-space formalism with spherical harmonic basis functions with accuracy to five decimal places. Comparison shows our present approach to be within $0.3 \%$ of the accurate value. It is interesting to note that due to the close approach of the spheres, the asymptotic value of $\bar{\varepsilon}$ in this case, $6.569 \varepsilon_{2}$, is about $60 \%$ larger than the ClausiusMossotti value of $4.1 \varepsilon_{2}$, where only the dipole contribution is accounted for.

(2) Orthorhombic array of closely spaced ellipsoids. Whereas the case of spherical inclusions may be accurately calculated by using an alternative method, the advantage of the present approach lies in its ability to handle arbitrary unit-cell microstructures. Accordingly, we next consider ellipsoids where the three semi-axes, $\alpha>\beta>\gamma$, are in the ratio of 1:0.8:0.7. The orthorhombic lattice constants $a_{1}, a_{2}, a_{3}$ in the three directions are 2.02,1.62, and 1.42, respectively, in units of $\alpha$. The Fourier transform of the characteristic function is given by

$$
\eta(\vec{k})=\frac{4 \pi \alpha \beta \gamma}{\kappa^{3} a_{1} a_{2} a_{3}}(\sin \kappa-\kappa \cos \kappa),
$$

where $\kappa=\sqrt{\left(\alpha^{2} k_{1}^{2}+\beta^{2} k_{2}^{2}+\gamma^{2} k_{3}^{2}\right)^{1 / 2}}$. The calculated $\bar{\varepsilon}_{11}$ $>\bar{\varepsilon}_{22}>\bar{\varepsilon}_{33}$ are shown in Fig. 1. Since the first moments of the spectral function are different along the three orthogonal directions, in contrast to the case of the spheres, they have to be evaluated from the characteristic function as

$$
\sum_{k \neq 0} \eta(-\vec{k}) \eta(\vec{k}) k_{1,2,3}^{2} / k^{2} .
$$

The results in the present case are 0.07564, 0.08412, and 0.09022 , respectively, along the $x(1), y(2)$, and $z(3)$ directions.

(3) Interpenetrating frames consisting of orthogonally intersecting rods with square cross sections. As both components percolate, the spectral function has a delta function

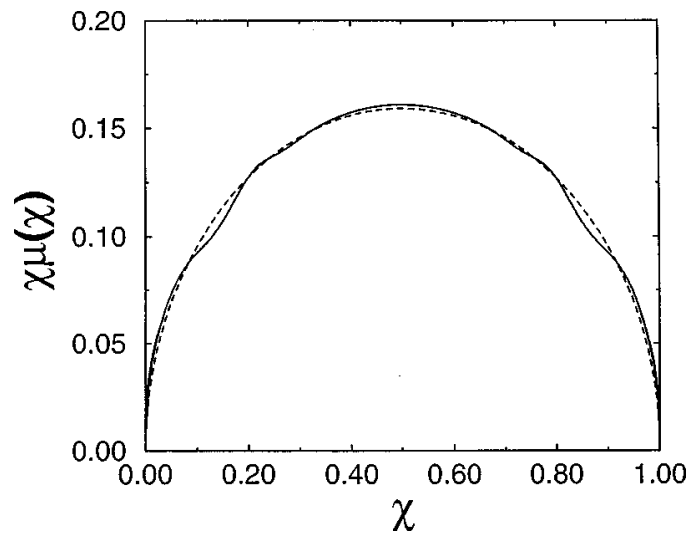

FIG. 2. The spectral function of the $2 \mathrm{D}$ checkerboard. The dashed line represents the analytical result.

contribution at $\chi=0$. The weight of this part, $C$, may be determined by the sum rule, since the part away from $\chi=0$ may be accurately evaluated from the recursive-FFT approach. For a simple cubic lattice with lattice constant $a$ and width of the rod $d=0.3 a$, the first moment, which is independent of $C$, can be evaluated as a function of $1 / N$. By extrapolating the results to $1 / N=0$, a value of 0.05650 is obtained (for $N=135$, the first moment has the value of 0.05566). This compares very favorably with the exact result of 0.056448 for $p=0.216$. The corresponding value of $C$ $=0.1034$. We find that high accuracy is generally possible for biconnected systems.

(4) The 2D checkerboard. The 2D checkerboard is a classical problem. Its self-dual property means the spectral function must have the symmetry $\chi \mu(\chi)=(1-\chi) \mu(1-\chi)$. In fact, the spectral function is known analytically: $\chi \mu(\chi)$ $=\sqrt{\chi(1-\chi)} / \pi,{ }^{12}$ thus it presents an opportunity to check our approach. In contrast to examples (1)-(3); the checkerboard is difficult in terms of obtaining accurate numerical results because of their "just touching" geometry. In this case, $\chi \mu(\chi)$ should have a power-law behavior in the neighborhood of $\chi=0$ and $\chi=1$ as deduced from the percolation theory. Accordingly, we use the recursive-FFT approach to calculate $\chi \mu(\chi)$, but replaces the result in the interval $0 \leqslant \chi$ $\leqslant A$ by $a \chi^{\alpha}(1+b \chi)$ (and similarly for the interval $1-A$ $\leqslant \chi \leqslant 1$ through the symmetry relation). The four parameters, $a, \alpha, b, A$ are to be optimally determined by the four requirements: the two sum rules plus the two continuity conditions for both the value and the first derivative of $\chi \mu(\chi)$. The results on the $2 \mathrm{D}$ checkerboard, with $\sim 10^{6}$ Fourier components, yield the exponent value $\alpha=0.5 \pm 0.05$, in good agreement with the analytical result. The resulting $\chi \mu(\chi)$ is compared with the exact expression (dashed line) in Fig. 2.

(5) The $3 D$ checkerboard. Again the spectral function should have the property that $\chi \mu(\chi)=(1-\chi) \mu(1-\chi)$. The Fourier transform of the characteristic function is given by

$$
\eta(\vec{k})=8 \frac{\sin \left(k_{1} a / 2\right) \sin \left(k_{2} a / 2\right) \sin \left(k_{3} a / 2\right)}{k_{1} k_{2} k_{3} a^{3}},
$$

where $a$ is the edge length of the cube, no analytical result is known as yet. But based on the assumption of edgeconduction dominance, it has been argued that asymptotically, the $\bar{\varepsilon}$ for the 3D checkerboard should have the same 


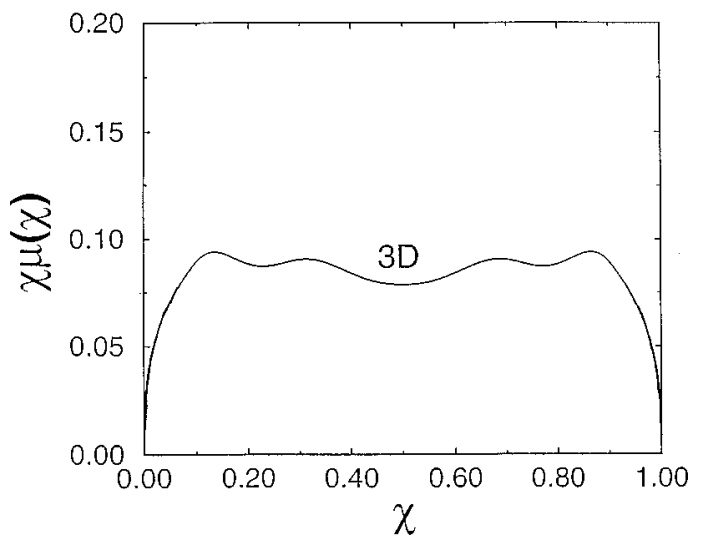

FIG. 3. The spectral function of the 3D checkerboard. The different heights of the $2 \mathrm{D}$ and $3 \mathrm{D}$ checkerboard spectral functions reflect the different normalization constants for the first moments, $p(1-p) / d$, where $d$ is the spatial dimensionality.

behavior as that for the 2D checkerboard, ${ }^{13}$ i.e., $\bar{\varepsilon}$ $\approx\left(\varepsilon_{1} \varepsilon_{2}\right)^{1 / 2}$ when $\left|\varepsilon_{1}\right| \gg\left|\varepsilon_{2}\right|$ or vice versa. The exponent value $\alpha$ of the $3 \mathrm{D}$ checkerboard has been calculated in the same manner as the 2D case. However, one million Fourier components in the present case means only 100 Fourier vectors in each direction, as opposed to 1000 Fourier vectors in the $2 \mathrm{D}$ case. In order to enhance accuracy, we calculated $\alpha$ as a function of $N$, and extrapolated the exponent value to $1 / N=0$. The result gives $\alpha=0.33$. That implies the maximum $N \sim 100$ used in the present calculation is insufficient for the accurate determination of the $3 \mathrm{D}$ checkerboard exponent at $\chi=0$. The $\chi \mu(\chi)$ for the 3D checkerboard is shown in Fig. 3. It would indeed be interesting to compare the 3D checkerboard result with a more rigorous solution of the electrostatic problem.

(6) In the last example, we use the present approach to calculate the $\bar{\varepsilon}$ of two random samples and check it against the experimentally measured results. The random samples consist of ten stainless steel spheres with a diameter of $1 \mathrm{~mm}$ dispersed in a cell with dimensions $5 \mathrm{~mm} \times 5 \mathrm{~mm} \times 1 \mathrm{~mm}$, filled with silicone oil $\left(\varepsilon_{2}=2.7\right)$. They are shown as insets to Fig. 4. The ac capacitance of the samples is measured with a HP 4284A Precision LCR meter. The capacitance of the cell is given by $C(\omega)=\varepsilon_{0} \bar{\varepsilon}(\omega) S / d$ (in MKS units), where $\varepsilon_{0}$ is the vacuum dielectric constant, $S$ is the area of the transverse

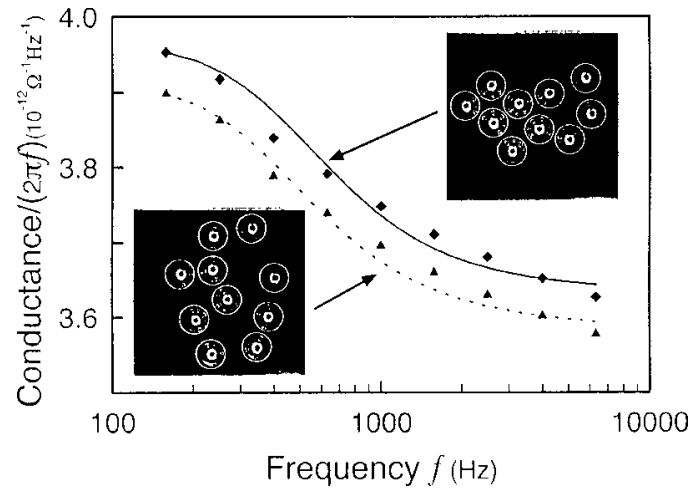

FIG. 4. Ac conductance plotted as a function of frequency. Diamonds and triangles represent experimentally data of the two random configurations shown as insets, measured along the horizontal direction. Calculated results are shown as solid and dashed lines. The calculated and measured effective dielectric constants are in the range of $30 i$ to $40 i$.

cross section of the cell $\left(5 \mathrm{~mm}^{2}\right)$, and $d$ the length of the cell $(5 \mathrm{~mm})$. The effective dielectric constant is calculated with the given microstructure as the unit cell of a periodic structure, in which the lattice constant in the thickness direction is purposely increased to $3 \mathrm{~mm}$ so as to minimize the interlayer interaction. The dielectric constant of the stainless sphere is given by $i \sigma / \varepsilon_{0} \omega$, with the conductivity $\sigma=1.1$ $\times 10^{4} \Omega^{-1} \mathrm{~cm}^{-1}$ determined by fitting one data point (at 150 $\mathrm{Hz}$ ) of the upper curve. ${ }^{14}$ Since the measured capacitance is almost purely imaginary, the results are plotted as ac conductance/ $\omega$. The calculation is done similar to the first example, with $\sim 10^{5}$ Fourier components. It is seen that the theory reproduces the experimental data quantitatively with excellent consistency. The lower curve, in particular, is calculated with no adjustable parameters.

\section{CONCLUDING REMARKS}

We have shown that the recursive-FFT approach, when coupled with the sum rules of the spectral function, can give accurate, efficient evaluation of the effective dielectric constant from arbitrary periodic microstructures. Application of the present approach to microtomography data is presently under way. It should be noted that after this manuscript was submitted, a similar work, utilizing the efficiency of FFT, was proposed by Moulinec and Suquet. ${ }^{15}$
*Permanent address: Dept. of Physics, Jiaotung University, Shanghai, People's Republic of China.

${ }^{1}$ J. W. S. Rayleigh, Philos. Mag. 34, 481 (1892).

${ }^{2}$ For the Clausius-Mossotti relation, see J. D. Jackson, Classical Electrodynamics, 2nd ed. (Wiley, New York, 1975), p. 155.

${ }^{3}$ D. Stauffer, Introduction to Percolation Theory (Taylor \& Francis, London, 1985).

${ }^{4}$ J. C. M. Garnett, Philos. Trans. R. Soc. London, Ser. A 203, 385 (1904).

${ }^{5}$ D. A. G. Bruggeman, Ann. Phys. (Leipzig) 24, 636 (1935).

${ }^{6}$ P. Sheng, Phys. Rev. Lett. 45, 60 (1980).

${ }^{7}$ For a generalized account, see A. N. Norris, A. J. Callegari, and P. Sheng, J. Mech. Phys. Solids 33, 525 (1985).

${ }^{8}$ B. P. Flannery, H. Deckman, W. Roberge, and K. D'Amico, Sci- ence 237, 1439 (1987); H. W. Deckman, K. L. D’Amico, J. H. Dunsmuir, B. P. Flannery, and S. M. Gruner, in Advances in X-ray Analysis, edited by C. S. Barrett, J. V. Gilfrich, R. Jenkins, T. C. Huang, P. K. Predecki (Plenum, New York, 1989), Vol 32, p. 641.

${ }^{9}$ D. J. Bergman and D. Stroud, Phys. Rep., Phys. Lett. 43C, 377 (1978); D. J. Bergman, Phys. Rev. B 19, 2359 (1979); G. W. Milton, Appl. Phys. A 26, 1207 (1981); G. W. Milton, J. Appl. Phys. 52, 5286 (1980).

${ }^{10}$ D. J. Bergman and K. J. Dunn, Phys. Rev. B 45, 13262 (1992).

${ }^{11}$ C. Lanczos, J. Res. Natl. Bur. Stand. 45, 225 (1950).

${ }^{12}$ J. B. Keller, J. Math. Phys. 5, 548 (1964); A. M. Dykhne, Zh. Eksp. Teor. Fiz. 59, 110 (1970) [Sov. Phys. JETP 32, 63 (1971)]. 
${ }^{13}$ J. B. Keller, J. Math. Phys. 28, 2516 (1987); M. Soderberg and G. Grimvall, J. Phys. C 16, 1085 (1983); G. Milton, Rep. Physics IV, Dept. Theoretical Physics, Univ. of Sydney, 1979.

${ }^{14}$ The fitted value of conductivity is in good agreement with 1.2 $\times 10^{4} \Omega^{-1} \mathrm{~cm}^{-1}$ given by the manufacturer (Goodfellow Stainless Steel 17-7PH).

${ }^{15}$ H. Moulinec and P. Suquet, Comput. Methods Appl. Mech. Eng. 157, 69 (1998). 